\title{
PREVALENCE OF ETIOLOGIES OF CHRONIC DIARRHEA AMONG PATIENTS ATTENDING ALEXANDRIA MAIN UNIVERSITY HOSPITAL
}

Hoda Abd Elhalim Elrefaay, Fahmy Helmy Hablas, Basma Elsayed Mahmoud Saeed

Department of Internal Medicine, Faculty of Medicine, University of Alexandria

\section{Introduction}

Chronic diarrhea is defined as an increase in the frequency of daily defecation ( 3 or more times daily) or increase in the amount of stool (more than $200 \mathrm{~g} /$ day of loose or liquid stools), which lasts more than 4 weeks. It was reported that almost $5 \%$ of the population at any given point in time suffered from chronic diarrhea, Although the exact prevalence is unknown. Diarrheal syndromes result from disturbances in any of the basic pathophysiological processes including osmosis, active secretion, exudation or inflammation, and altered motility. Most etiologies will have a complex pathophysiology involving one or more of these mentioned mechanisms.

Diagnosis of chronic diarrhea depends on detailed history, physical examination, laboratory investigations, imaging and endoscopic procedures.

\section{Aim of the work}

To provide the prevalence of different etiological causes of chronic diarrhea among the patients attended Alexandria Main University Hospital (AMUH).

\section{Subjects and Methods}

\section{Subjects:}

- This study included Egyptian adult Patients who complained of chronic diarrhea and were attending Gastroenterology clinic in AMUH in the period from Augus 2019 to August 2020.

\section{Methods}

All patients were subjected to the following:

- Detailed history taking with emphasis on: age, duration, pattern, timing, relation to food, family history, weight loss, history of travel, history of fever, history of previous surgery or systemic disease, history of drugs including laxatives or alcohol abuse and history of radiation or chemotherapy therapy.
Thorough systemic Physical examination including:

- Findings of dehydration such as dry mucus membranes and decreased skin turgor

- Eye and neck examination with stress on the presence episcleritis, exophthalmosis, goiter and Lymphadenopathy.

- Abdominal examination with stress on the presence or absence of bowel sounds, abdominal distention, localized or generalized tenderness, masses, cutaneous fistula, and palpable organs.

- Rectal examination with stress on the presence of palpable rectal mass or perianal fistula associated with Crohn's disease.

- Skin examination such as the presence of dermatitis herpetiform, erythema nodosum, pyodermagangrenosum, and Psoriasis

- laboratory investigations including CBC, ESR, CRP, liver function tests, renal function tests, sugar profile, thyroid profile, Stool analysis, H.pylori S antigen in stool, Fecal calprotectin, FIT, tTG IgA, IgG, Fecal elastase, HIV antibodies and PCR for lactose intolerance. Also EGD \& duodenal biopsy and Ileocolonoscopy. After exclusion of organic diseases and depending on Rome
IV criteria, Diagnosis of IBS- diarrhea was established.

\section{Results}

Table : Prevalence of etiologies of chronic diarrhea among patients attending GIT clinic at AMUH

\begin{tabular}{|l|c|c|}
\hline Etiologies & No. & \% \\
\hline IBD (CD) & 27 & 39.1 \\
\hline IBD (UC) & 20 & 29.0 \\
\hline IBS-D & 10 & 14.5 \\
\hline Cancer colon & 5 & 7.2 \\
\hline Celiac disease & 3 & 4.3 \\
\hline Microsopic colitis (Lymphocytic type) & 2 & 2.9 \\
\hline Lactose intolerance & 2 & 2.9 \\
\hline
\end{tabular}

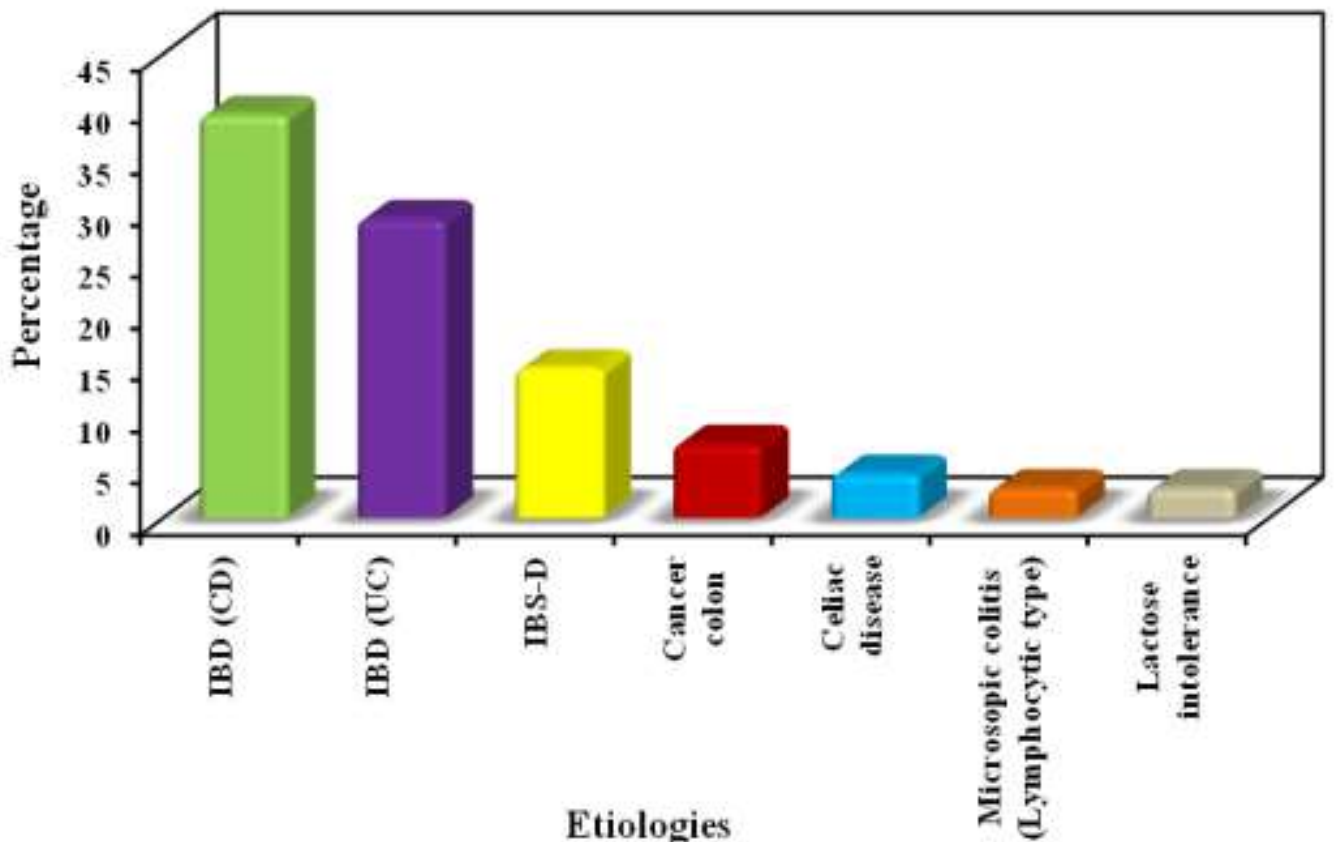

Figure : Prevalence of etiologies of chronic diarrhea among patients attending GIT clinic at AMUH $(\mathrm{n}=69)$

\section{Conclusion}

- Among 69 studied patients who were attended Gastroenterology clinic in AMUH in the period from August 2019 to August 2020, the most common etiology was IBD $(68.1 \%),(39.1 \%)$ patients were CD and (29.0\%) pants were UC, secondly IBS-D (14.5\%), then cancer colon (actose intorance an microscopic colitis $(2.9 \%)$

$$
\text { Macsing }
$$

